\title{
Traduire
}

Une autre perspective sur rit traduction

Revue française de la traduction

$241 \mid 2019$

La formation à l'honneur

\section{Pour une pédagogie de la textualisation}

Verbaliser la reverbalisation, construire la communication, affirmer le traducteur

\section{Ludovica Maggi}

\section{Q OpenEdition}

1 Journals

Édition électronique

URL : http://journals.openedition.org/traduire/1854

DOI : $10.4000 /$ traduire.1854

ISSN : 2272-9992

Éditeur

Société française des traducteurs

Édition imprimée

Date de publication : 16 décembre 2019

Pagination : 71-80

ISSN : 0395-773X

\section{Référence électronique}

Ludovica Maggi, « Pour une pédagogie de la textualisation », Traduire [En ligne], 241 | 2019, mis en

ligne le 16 décembre 2019, consulté le 10 décembre 2020. URL : http://journals.openedition.org/ traduire/1854; DOI : https://doi.org/10.4000/traduire.1854 


\section{Pour une pédagogie de la textualisation}

Verbaliser la reverbalisation, construire la communication,

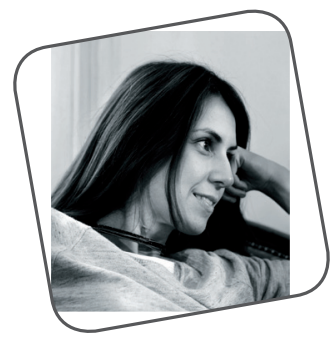
affirmer le traducteur.

\section{Ludovica Maggi}

\section{Introduction}

Dans ce numéro consacré à la formation, nous souhaitons soumettre à l'attention des collègues traducteurs, chercheurs et formateurs une réflexion de fond sur l'étendue de l'objet de la traduction, suggérée par l'observation d'un comportement traductif qui nous apparaît comme fréquent chez les apprenants: la résistance à la reformulation active du texte cible pensé en tant que structure organique et intégrée, supérieure à l'assemblage par juxtaposition d'une série de segments traductifs cibles.

Ce faisant, nous espérons ouvrir la voie vers un traitement descriptif et analytique de ce phénomène au sein de la communauté traductologique, ainsi que vers l'élaboration d'une méthode pédagogique davantage centrée sur la sensibilisation au texte.

Dans cette optique, nous suggérons la prise en compte du texte comme unité traductive de destination et le recours à la catégorie traductologique de la textualisation pour consolider la phase scripturale de l'opération traductive. 
À cet effet, nous rappelons l'intérêt d'une interaction plus assumée de la traductologie et de la pédagogie de la traduction avec la linguistique textuelle, à étendre à l'analyse du discours et aux théories de la communication.

Si cette réflexion veut avoir un impact sur la pédagogie de la traduction, prônant la mise en place d'un cadre plus systématisé pour l'apprentissage de la reverbalisation et, partant, une amélioration de la qualité des traductions produites par les étudiants, elle ose également suggérer la valeur pragmatique d'une formation à la textualisation en vue du positionnement des jeunes traducteurs sur le marché du travail.

\section{Les apprenants et le tabou du texte: de la reverbalisation à la textualisation}

Au cours de cinq années de pratique de l'enseignement de la traduction au sein du master en communication interculturelle et traduction de I'ISIT, nous avons eu l'occasion de constater que le remaniement de la structure de la matière textuelle source lors de la génération du texte cible est souvent freiné par une sorte d'interdit non avoué, une réticence à l'intervention scripturale, un tabou de la construction active du texte traduit.

Nous sommes là en présence d'un phénomène qui s'apparente, nous semble-t-il, à la difficulté, connue chez les apprenants, d'exercer - après la déverbalisation - une reverbalisation adéquate, c'est-à-dire d'exprimer le sens saisi dans une forme convenable à la langue-culture d'arrivée (Seleskovitch et Lederer, 2015/1984).

Formulé volontairement de façon générale, aux antipodes des instructions comparatistes chères aux adeptes des versions et libres de toute référence aux catégories linguistiques, le principe de la reverbalisation n'est pas, en soi, des plus aisés à transmettre: le maître traducteur l'explique le plus souvent à son apprenti en l'invitant à renoncer aux calques lexicaux et syntaxiques et à rechercher en revanche la fluidité, le naturel, les idiomatismes propres à la langue de destination, pour que le texte traduit «ne sente pas la traduction». Armés de bonne volonté, les étudiants se prêtent à l'exercice, avançant par tâtonnements et réussissant enfin, plus ou moins tôt dans leur apprentissage, à pétrir de façon 
autonome la matière traductive cible, reformulant enfin «comme cela se doit» l'unité de sens qui en constitue le point de départ.

Or, un obstacle s'interpose à la réalisation d'une traduction «fluide», telle qu'exigée par le grand principe de la reverbalisation. Il s'agit précisément, à notre sens, de l'étendue de la portion de texte sur laquelle les apprenants se sentent autorisés à exercer ladite reverbalisation.

En effet, si les jeunes traducteurs élargissent progressivement leur périmètre de traitement, s'éloignant très tôt du mot à mot et apprenant au fil du temps à rechercher l'autonomie lexicale et syntaxique de leur production cible, tout en respectant la contrainte de la terminologie spécialisée, l'affranchissement de la formulation source semble procéder le plus souvent par blocs isolés. Ainsi, la phrase, le paragraphe tout au plus, s'impose comme unité de sens maximale, le processus recommençant au bloc suivant, avec, pour résultat, une somme d'unités cibles autarciques, et un niveau de raccordement et d'harmonisation faible ou insuffisant.

Cette pratique suscite une double exigence: d'une part la description et la compréhension correcte du phénomène; d'autre part la mise au point d'instruments théoriques efficaces permettant de sensibiliser les apprenants à son émergence et de les aider à le contraster.

S'agissant du premier point, notre ressenti est que la difficulté éprouvée par les étudiants porte sur l'identification de la délimitation de l'unité traductive et concerne le deuxième volet de l'opération traductive (la restitution de la source en langue cible) plus encore que le premier (l'appréhension de la source).

Autrement dit, les étudiants en traduction ne semblent pas prendre suffisamment en compte la dimension du texte source: trop peu sensibles aux éléments linguistiques qui déterminent sa structure et son agencement, ils ne les identifient pas comme porteurs de sens et ne s'interrogent pas sur les implications que peut avoir sur la traduction le déploiement de ces traits dans le cadre de destination.

En termes théoriques, cela impliquerait de définir, parallèlement à l'unité de sens, une unité de reverbalisation, puis de s'interroger sur son étendue, envisageant qu'elle puisse atteindre jusqu'à la totalité du texte cible. 
Plus encore, il serait possible d'imaginer un degré supérieur de la reverbalisation, qui pourrait se nommer textualisation: si la première concerne la transformation de l'unité de sens source en unité de formulation cible, la deuxième se rapporterait à l'ensemble des unités de formulation cible, représentant plus que la somme de chacune d'entre elles. La textualisation serait alors la mise en système et en écho des différentes unités, visant à constituer un tissu cible dans le respect des normes du comportement textuel propre, de façon large, à la langue-culture cible et défini de manière plus circonstanciée par des paramètres tels que le genre du texte, la situation et la visée communicative d'arrivée.

II y aurait ainsi lieu de redoubler, pour l'unité traductive cible, l'exercice d'étirement collectivement suggéré pour l'unité traductive source (Équivalences, 45/1-2, 2018), tout en adoptant une perspective fonctionnaliste, c'est-à-dire en admettant que les normes qui régissent les dynamiques textuelles sont tout autres qu'universelles et que le travail de reverbalisation doit prendre en compte les leviers du texte au même titre que les autres catégories linguistiques.

\section{Le texte: lieu de convergence entre approche linguistique et théorie du sens, pour la traduction}

Aborder le texte comme unité traductive de destination et la textualisation comme catégorie traductologique implique tout naturellement l'adhésion à la préconisation de Mona Baker (1992), qui recommande de faire progresser l'équivalence du mot au texte.

En ce sens, il convient de revaloriser les axes de réflexion de Baker, principalement centrés sur le thème-rhème, la cohésion et la cohérence. Plus encore, il nous semble pertinent de les intégrer dans le cadre d'un plus ample réseau de théories et de catégories de la linguistique textuelle.

Ainsi, nous confirmons l'intérêt de la perspective fonctionnelle de la phrase (de Jan Firbas, 1964 à Martin Adam, 2007), qui rappelle à quel point l'ordre de présentation des informations pèse dans le transfert du sens et suggère, en perspective traductologique, au moins deux points de vigilance: 1) la 
dynamique du connu et du nouveau n'est pas confinée à la phrase, mais se propage dans la succession de phrases, de sorte que la formulation doit constamment être surveillée, pour ne pas surprendre le lecteur avec une information nouvelle qui ne serait pas linguistiquement signalée comme telle ou, au contraire, le gêner en lui soumettant comme ex novo des éléments de sens qu'il aurait déjà acquis; 2) les modalités de hiérarchisation linguistique de l'information varient selon les langues-cultures, les genres textuels, les contextes de communication, les auteurs; ils sont donc susceptibles de nécessiter un recalibrage lors de la constitution du texte cible au moyen d'une langue-culture autre, possiblement dotée d'une autre culture des genres, ou employée dans un contexte de communication légèrement glissant par rapport à celui qui a présidé à la conception du texte source.

Nous faisons également référence aux grands fondements de la discipline posés par de Beaugrande et Dressler (2013/1981): la cohésion et la cohérence certes, mais également l'intentionnalité, l'acceptabilité, l'informativité, la situationnalité et l'intertextualité. En effet, ces catégories révèlent au traducteur la fine grille d'interconnexions qui structure le texte, rendant manifeste la fonction de véhicule de sens remplie par l'architecture physique qu'est la langue dans son déploiement textuel. Comme pour la dimension fonctionnelle de la phrase, ces principes acquièrent une valeur traductologique dès lors qu'ils guident l'analyse de l'unité traductive source, puis la recomposition de l'unité traductive cible dans le respect des exigences propres au cadre de destination.

Surtout, nous reconnaissons l'intérêt, pour la traduction, des études de linguistique textuelle de Jean-Michel Adam (2005), qui marquent de façon explicite la relation entre la description du texte en tant que système et la constitution progressive du sens par ce même système. Nous pensons notamment à l'anaphore et la cataphore comme dispositifs de coréférence non simplement lexicale, mais sémantique; à la progression informative, sorte d'effet domino correctif qui dessine la pensée au cours du texte; à la différence entre le présupposé, impliqué par la structure linguistique, et le préconstruit notionnel attendu chez le lecteur; à la prise en charge énonciative, directe ou médiatisée, et à l'orientation argumentative; aux organisateurs qui balisent le texte 
et signalent l'agencement spatial et temporel, la présence d'une série, l'ajout ou la clôture, le changement de topicalisation, l'exemplification.

Enfin, c'est en suivant le devenir du sens dans ce creuset d'articulations qu'est le texte, qu'il nous semble possible d'envisager une dynamique de convergence entre la linguistique et la réflexion traductologique inhérente à la théorie interprétative de la traduction ( TIT), pourtant programmatiquement fondée sur une émancipation de la linguistique. De telles retrouvailles seraient aussi vertueuses que souhaitables, en ceci qu'elles rendraient possible une «verbalisation» de la reverbalisation: une fois nommés, les nœuds - linguistiques et textuels - du sens deviendraient plus facilement identifiables; l'acte intuitif de la reformulation deviendrait plus structuré, plus transparent, sa transmission pédagogique plus aisée.

\section{Dans le texte et au-delà : discours et communication}

En plaidant en faveur d'un recentrement de la pédagogie de la traduction autour du texte, nous avons jusqu'ici souhaité souligner les apports potentiels d'une plus grande sensibilité à la linguistique textuelle en faveur d'une reverbalisation réussie. Nous avons ainsi prôné un rapprochement entre linguistique et TIT, rappelant le rôle du texte en tant que véhicule de sens.

Or la relation texte-sens n'est pas binaire. Ces deux dimensions ne peuvent se rejoindre que par le biais d'un tertium, clairement identifié par Jean-Michel Adam (2005), Iorsqu'il choisit pour son ouvrage La Linguistique textuelle le soustitre Introduction à l'analyse textuelle des discours.

C'est bien le discours qui justifie, valorise et informe le travail de décodage et de réencodage textuel en traduction. C'est d'ailleurs sûrement dans cet espace conceptuel - où le texte se fait acte, idée, position, relation, évocation d'autres actes, idées, positions, relations - qu'il convient de placer le dernier volet de la réflexion de Baker (1992), relatif à la prise en compte de la dimension pragmatique.

Pour une reverbalisation assumée, il serait alors également souhaitable que les apprenants se familiarisent avec les principes de l'analyse du discours. 
En deçà des enjeux idéologiques et politiques de la parole, l'exercice de la traduction mériterait en effet de se fonder sur la connaissance d'un faisceau de cadres intellectuels relatifs au déploiement textuel et contextuel de la langue, tels que la théorie des actes linguistiques d'Austin (1970/1962); celle des genres discursifs de Bakhtine (1984), dont Eggins (2004) ne manque pas de préciser le caractère culturel; ou encore celle des fonctions sémiotiques d'Halliday (1978), qui avance une description du discours en termes de champ (le sujet abordé), de mode (l'articulation du réseau textuel) et de teneur (la relation interpersonnelle construite à travers le texte). Un éclairage précieux serait aussi apporté par certains écrits fondamentaux de Patrick Chareaudeau: sa thèse sur les conditions linguistiques de l'analyse du discours (1977), ou ses travaux portant sur le contrat de communication et les genres discursifs, les stratégies de discours comme processus énonciatifs, le contexte et la situation de communication (Chareaudeau, 1994, 1999, 2000).

À la recherche des clés de la reverbalisation du sens, nous avons jusqu'ici invoqué le terrain du texte, puis celui, plus large, du discours. Si la sensibilisation à ces deux aspects nous apparaît comme le minimum nécessaire pour un exercice avisé de la reverbalisation, cette dernière ne peut que bénéficier d'un élargissement du périmètre des compétences du traducteur.

Le discours, une fois assimilé à une organisation textuelle de la pensée, peut alors être envisagé comme une position idéologique, individuelle ou collective, sur un thème donné - avec une démarche qui peut impliquer l'intertextualité -, voire comme espace de déploiement de positions de force réciproques, sur la base d'une approche critique de l'analyse du discours (van Dijk, 1993, entre autres). Dans un cadre plus large, il serait également possible d'éclairer l'action traductive par un regard sur les dynamiques communicationnelles, dont la traduction est à la fois un véhicule et un cas d'espèce. Enfin, au-delà des confins du texte, là où cela se révèle pertinent, il est possible d'envisager - comme nous l'avons fait par ailleurs (Maggi, 2019) - une réflexion traductologique sur l'œuvre en tant que construction idéique, à la fois interprétée et façonnée par la traduction. 


\section{Pistes}

Au terme de cette brève réflexion sur les dimensions théoriques qui pourraient alimenter une meilleure compréhension de l'articulation du texte et, par ce biais, une meilleure reformulation de ce dernier, se pose la question de connaître l'ampleur et les modalités de transmission les plus adéquates des principes linguistiques et discursifs pertinents.

Renvoyer les apprenants à des lectures théoriques ou prévoir des cours de linguistique textuelle, d'analyse du discours et de communication au sein des cursus en traduction semblent être des solutions utiles et viables. Toutefois, il convient de repenser ces matériaux théoriques pour les mettre plus spécifiquement au service de la traduction et de sa pédagogie. Un travail est donc à faire en termes de recherche empirique, d'une part pour vérifier l'intérêt réel, aux fins de la reverbalisation, des classes conceptuelles évoquées: quelles catégories de difficultés rencontrent précisément les étudiants? Pourquoi? Avec quelle fréquence? Quels leviers permettraient de les résoudre, ou de les prévenir? D'autre part, sur le front de la théorisation, des cadres ad hoc sont à élaborer pour préciser l'applicabilité traductologique des modèles linguistiques et discursifs existants et/ou pour identifier de nouveaux cadres, propres à l'exercice traductif. Enfin, les méthodes d'enseignement de la traduction textuelle méritent d'être affinées.

À cet effet, I'on pourrait repartir du travail de Baker - récemment mis à jour (Baker, 2011) - pour en concevoir un complément ou une alternative: un ouvrage consacré à l'étude des enjeux textuels, discursifs, voire communicationnels, de la traduction, qui serait plus efficace si l'on veillait à estomper la marque de la pédagogie comparatiste des langues - si présente dans le manuel de Baker - et à rendre sa lecture abordable davantage aux étudiants qu'aux experts.

Cet effort de recherche, de théorisation et de pédagogie aurait une valeur ajoutée considérable pour la discipline et la profession, dans un contexte où la formation en traduction doit plus que jamais être légitimée aux yeux du public et peut-être même de ceux qui la choisissent.

En effet, savoir reconnaître et décliner culturellement les pivots du texte et, partant, du discours et de la communication est aujourd'hui crucial dans une industrie de la traduction 
fortement marquée par l'intelligence artificielle. D'une part, les compétences textuelles et discursives permettent au traducteur d'intégrer avec pertinence la chaîne de valeur de la traduction automatique (TA) - et ce, en intervenant en aval sur une post-édition de qualité ou en interagissant en amont avec les chercheurs et les linguistes informaticiens pour faire évolver l'apprentissage par la machine du stade de la langue (niveau auquel la TA agit encore à présent) à celui du texte et du discours. D'autre part, ces mêmes compétences restent la clé d'une activité traductive humaine et distinctive dans des domaines porteurs comme la communication de marque, la traduction pour le doublage ou la bande dessinée, sans oublier des terrains plus traditionnels tels que la traduction éditoriale, théâtrale ou journalistique.

Enfin, dans une perspective sociologique, la construction d'une conscience traductive et traductologique du texte et du discours ouvre la voie à l'affirmation du traducteur comme sujet du texte et de la communication. Défini comme décideur et créateur de la matière textuelle cible par les acteurs mêmes de la formation, l'apprenant se sentirait autorisé à se percevoir et à se présenter comme tel, se libérant ainsi de sa réticence opérationnelle et œuvrant, stratégiquement, pour un positionnement professionnel davantage centré sur l'action, la visibilité, la responsabilité et le leadership.

\section{I.maggi@isit-paris.fr}

Diplômée en lettres classiques et docteur en traductologie, Ludovica Maggi est interprète de conférence et traductrice, spécialisée dans le discours institutionnel et la communication d'entreprise. Directrice académique du master en communication interculturelle et traduction à I'ISIT, elle enseigne dans cette même école la traduction, les théories de la traduction et de la communication et I'histoire de la traduction. Ses recherches portent sur la dimension communicative de la traduction, la traduction de l'oralité et la réception du passé dans les discours traductif et communicationnel 
ADAM Jean-Michel, La Linguistique textuelle. Introduction à l'analyse textuelle des discours, Paris, Armand Colin, 2005.

ADAM Martin, A handbook of functional sentence perspective, Brno, Masarykova Univerzita, 2007.

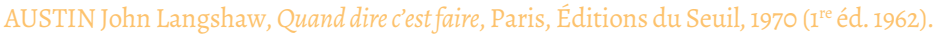

BAKHTINE Michail, Esthétique de la création verbale, Paris, Gallimard, 1984.

BAKER Mona, In Other Words, Londres, Routledge, 1992.

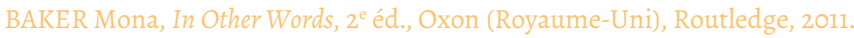

CHAREAUDEAU Patrick, Des conditions linguistiques d'une analyse du discours, thèse de doctorat, Paris, Sorbonne, 1977.

CHAREAUDEAU Patrick, "D’une théorie du langage à l'analyse du discours», in Connexions, 38, 1982 .

CHAREAUDEAU Patrick «Catégories de langue, catégories de discours et contrat de communication", in MOIRAND Sophie et al., Parcours linguistiques de discours spécialisés, Berne, Peter Lang, 1994.

CHAREAUDEAU Patrick, Une problématique des genres. De la situation à la construction textuelle, 1999, www.patrick-charaudeau.com/Une-problematique-des-genresDe-la.html, consulté le 21 octobre 2019.

CHAREAUDEAU Patrick, «De la compétence situationnelle aux compétences de discours ", in Actes du colloque de Louvain-la-Neuve sur Compétence et didactique des langues, 2000, www.patrick-charaudeau.com/De-la-competence-situationnelle.html, consulté le 21 octobre 2019.

DE BEAUGRANDE Robert et DRESSLER Wolfgang, Introduction to Text Linguistics,

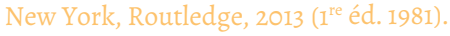

EGGINS Suzanne, An Introduction to Systemic Functional Linguistics, New York, Continuum, 2004.

FIRBAS Jan, «On defining the theme in functional sentence analysis», Travaux linguistiques de Prague, 1, 1964, p. 267-280.

FROELIGER et al. (dir.), Des unités de traduction à l'unité de la traduction, in Équivalences, 45/1-2, 2018.

HALLIDAY Michael A. K., Language as Social Semiotic. The Social Interpretation of Language and Meaning, Londres, Edward Arnold, 1978.

MAGGI Ludovica, Herméneutique, oralité, temporalité. L'écriture traductive théâtrale de l'interprétation des classiques à la mise en voix, thèse de doctorat, Paris, La Sorbonne Nouvelle, 2019.

SELESKOVITCH Danica et LEDERER Marianne, Interpréter pour traduire, Paris, Les Belles Lettres, 2015 (1 $1^{\mathrm{re}}$ éd. 1984).

VAN DIJK Teun A., «Principles of critical discourse analysis», in Discourse \& Society, 2, 1993, p. 249-283. 